\title{
Temperature Prediction of Photovoltaic Panels Based on Support Vector Machine with Pigeon-Inspired Optimization
}

\author{
Siyuan Fan, ${ }^{1}$ Shengxian Cao $\mathbb{D}^{1},{ }^{1}$ and Yanhui Zhang $\mathbb{D}^{2}$ \\ ${ }^{1}$ School of Automation Engineering, Northeast Electric Power University, Jilin 132012, China \\ ${ }^{2}$ Shenzhen Institutes of Advanced Technology, Chinese Academy of Sciences, Shenzhen 518055, China \\ Correspondence should be addressed to Shengxian Cao; csxlb_jl@163.com and Yanhui Zhang; zhangyh@siat.ac.cn
}

Received 15 June 2020; Revised 4 August 2020; Accepted 16 November 2020; Published 18 December 2020

Academic Editor: Zong Woo Geem

Copyright (c) 2020 Siyuan Fan et al. This is an open access article distributed under the Creative Commons Attribution License, which permits unrestricted use, distribution, and reproduction in any medium, provided the original work is properly cited.

The output stability of the photovoltaic (PV) system is directly affected by temperature change of PV panels. In this paper, a novel temperature prediction method of PV panels with support vector machine (SVM) is proposed, which can solve the temperature prediction problem in a complex environment. In order to optimize parameters of SVM, a Pigeon-Inspired Optimization (PIO) method is given. Meanwhile, the delay factor (DF) is added to improve the PIO algorithm for avoiding the problem of local optimum. Moreover, a multisensor monitoring system of PV is established, and the collected data of temperature are used to train and verify the accuracy of the model. Finally, the proposed method is evaluated using synthetic and actual data sets. Simulation results show that the DFPIO-SVM can obtain better predictive performance.

\section{Introduction}

Due to the shortage of energy, emission of pollution, and increasing demand, people have to seek new energy to replace traditional fossil energy. Solar energy has been attracting more and more attention because it has the advantages of being green, clean, renewable, and so on. However, in natural conditions, the power generation performance of PV panels is affected by various factors, especially, the temperature change of PV panels $[1,2]$. As one of the most relevant factors, the temperature of $\mathrm{PV}$ panels has attracted wide attention in recent years [3, 4]. When the temperature of PV panels rises by $1^{\circ} \mathrm{C}$, the efficiency of power generation decreases by about $0.22 \%$ [5]. Therefore, during the operation and maintenance of PV power stations, how to accurately predict the temperature of $\mathrm{PV}$ panels becomes an interesting problem.

The temperature of PV panels is often affected by natural factors such as irradiation, ambient temperature, wind speed, and dust $[6,7]$. With the increase of radiation intensity and ambient temperature, the temperature of PV panels will increase accordingly. For every $1^{\circ} \mathrm{C}$ increase of the ambient temperature, the temperature of $\mathrm{PV}$ panels will increase by $0.851^{\circ} \mathrm{C}[8,9]$. Due to the influence of wind, the temperature of PV panels will decrease faster. For every $1 \mathrm{~m} /$ $\mathrm{s}$ increase of wind speed, the temperature of PV panels will decrease by $0.421^{\circ} \mathrm{C}$ [10]. In order to better understand the temperature change rule of $\mathrm{PV}$ panels, many effective prediction models have been proposed by researchers. Almaktar et al. [11] proposed a novel temperature prediction model of PV panels based on the collected data in a tropical climate, which has better results. Barykina and Hammer [12] proposed a Faiman temperature model of PV panels, which evaluated four different panels in outdoor conditions with different climates. Muzathik [13] proposed a simple temperature prediction model of PV panels based on the environmental variables. Ceylan et al. [14] established a temperature prediction model of PV panels based on the backpropagation (BP) neural network by using different ambient temperature and radiation intensity data. However, these methods need to consider as many factors as possible, but the complex field environment makes it difficult to accurately measure some parameters, so a good prediction effect cannot be obtained.

With the development of artificial intelligence technology, a lot of machine learning algorithms, Long Short- 
Term Memory (LSTM) [15-17], BP [18, 19], SVM [20, 21], Radial Basis Function Neural Network (RBFNN) [22-24], Echo State Network (ESN) [25-27], and Deep Belief Network (DBN) [28], have been widely used in time series prediction. SVM is used to solve regression problems due to its advantages of no large amount of sample data and fast training speed. Selakov et al. [29] proposed a novel hybrid model based on PSO-SVM, which is applied to short-term electrical load prediction. Zhang et al. [30] proposed a load prediction method based on SVM with dragonfly algorithm (DA) in microgrid. For the unpredictability and periodicity of power load, Ma et al. [31] investigated a prediction model based on SVM with a modified firefly algorithm (MFA). Lu [32] proposed surface roughness prediction methods based on SVM with the artificial bee colony $(\mathrm{ABC})$ optimization algorithm in the manufacturing process.

Since the selection of key parameters of SVM has a great influence on the accuracy of the network model, some swarm intelligence optimization algorithms, including $A B C$ [33, 34], PSO [29, 35, 36], Artificial Fish Swarms Algorithm (SFSA) [37], and Differential Evolution (DE) [38, 39], are applied to optimize these parameters by many researchers. The basic idea of these algorithms is to simulate the behavior of biological groups in nature, in which its own scalability, robustness, and flexibility are relatively strong $[40,41]$. In 2014, Duan and Qiao [42] proposed a new swarm intelligence optimization algorithm, namely, Pigeon-Inspired Optimization, inspired by pigeon homing behavior in nature. PIO has been widely used in UAV formation $[43,44]$, control parameter optimization [45-47], image processing [48], and life science $[49,50]$, and it has achieved good results in experimental applications.

In this paper, a temperature prediction method of $\mathrm{PV}$ panels based on SVM with PIO is introduced. Firstly, on the basis of analysing the SVM principle, the PIO algorithm is adopted to optimize the SVM parameters, and the PV panels temperature prediction model is established. And then, according to the characteristics of particle velocity change before and after two times, DF is introduced into the PIO algorithm to improve the search accuracy. Finally, in order to verify the prediction performance of DFPIO-SVM, for the temperature data of PV panels from the distributed PV power station, the comparison results of different optimization methods are given.

The remainder of this paper is as follows: a multisensor information fusion (MSIF) monitoring system is introduced in Section 2. The principle of SVM and three comparison optimization methods are given in Section 3. The DFPIO-SVM prediction model is proposed in Section 4. The simulation example results are performed in Section 5 .

\section{MSIF Monitoring System}

In this section, we will introduce a MSIF monitoring system of PV station, which can collect and record data of current, voltage, temperature of PV panels, and meteorological information.

2.1. The Structure of Monitoring System. The MSIF monitoring system consists of a field data acquisition system, a wireless data transmission system, and the software management system. In Figure 1, the data information from monitoring point $A$ and point $B$ is transmitted to monitoring point $C$ and then to the monitoring center. Meanwhile, each monitoring point can also be used as a relay system. The transmission distance between points can be $5 \mathrm{~km}$. The temperature of PV panels is transmitted to the monitoring center by the wireless system, which can provide accurate real-time information for the operating and maintenance personnel.

The field data acquisition system includes a meteorological monitoring system, temperature sensor, data transmitter, and other equipment. Solar irradiance, ambient temperature and humidity, wind speed, wind direction, and atmospheric pressure obtained from sensors are transmitted to a data monitoring host. The digital DS18B20 temperature sensor is used to measure the temperature of PV panels. By using the characteristics of a sensor single bus, complex signal transmission lines are avoided. When measuring the temperature of PV panels, it is necessary to make a measurement of different positions, and the average temperature $T_{a}$ is expressed as

$$
T_{a}=\frac{T_{1}+T_{2}+T_{3}+T_{4}}{4},
$$

where $T_{a}$ denotes the average temperature and $T_{1,2,3,4}$ denotes the temperature of different positions.

2.2. The Data Source. The experimental site is a distributed PV power station in western Jilin, China. The area is located at $\mathrm{N} 45^{\circ} 31^{\prime}$ and $\mathrm{E} 122^{\circ} 44^{\prime}$. The power station has an output of $12 \mathrm{MW}$. The annual sunshine duration is 1300 hours and the average annual power generation is 15.8 million KWh. The PV power station has 46,200 PV panels, which are oriented toward the south. The angle of inclination to the ground is $43^{\circ}$, and the tilt angle of the fixed bracket is not adjustable. Two PV panels are installed side by side and the dimension is $1640 \mathrm{~mm} \times 990 \mathrm{~mm} \times 35 \mathrm{~mm}$. The MSIF monitoring system at the experimental site is shown in Figure 2.

2.3. Recorded Temperature Data of PV Panels. The data in this paper were obtained through the MSIF monitoring system established in a PV power station. The monitoring system recorded the temperature, meteorological information, voltage, current, and other data of the PV panels every 15 minutes. The prediction model is trained and validated using 3000 data samples from January to June 2018 under different weather conditions.

The open-circuit voltage decreases PV panels with the increase of temperature, and the voltage temperature coefficient is $-(210 \sim 220) \mathrm{mV} /{ }^{\circ} \mathrm{C}$. Similarly, the peak power decreases PV panels with the temperature increase, which directly affects the generation efficiency of PV systems. As 


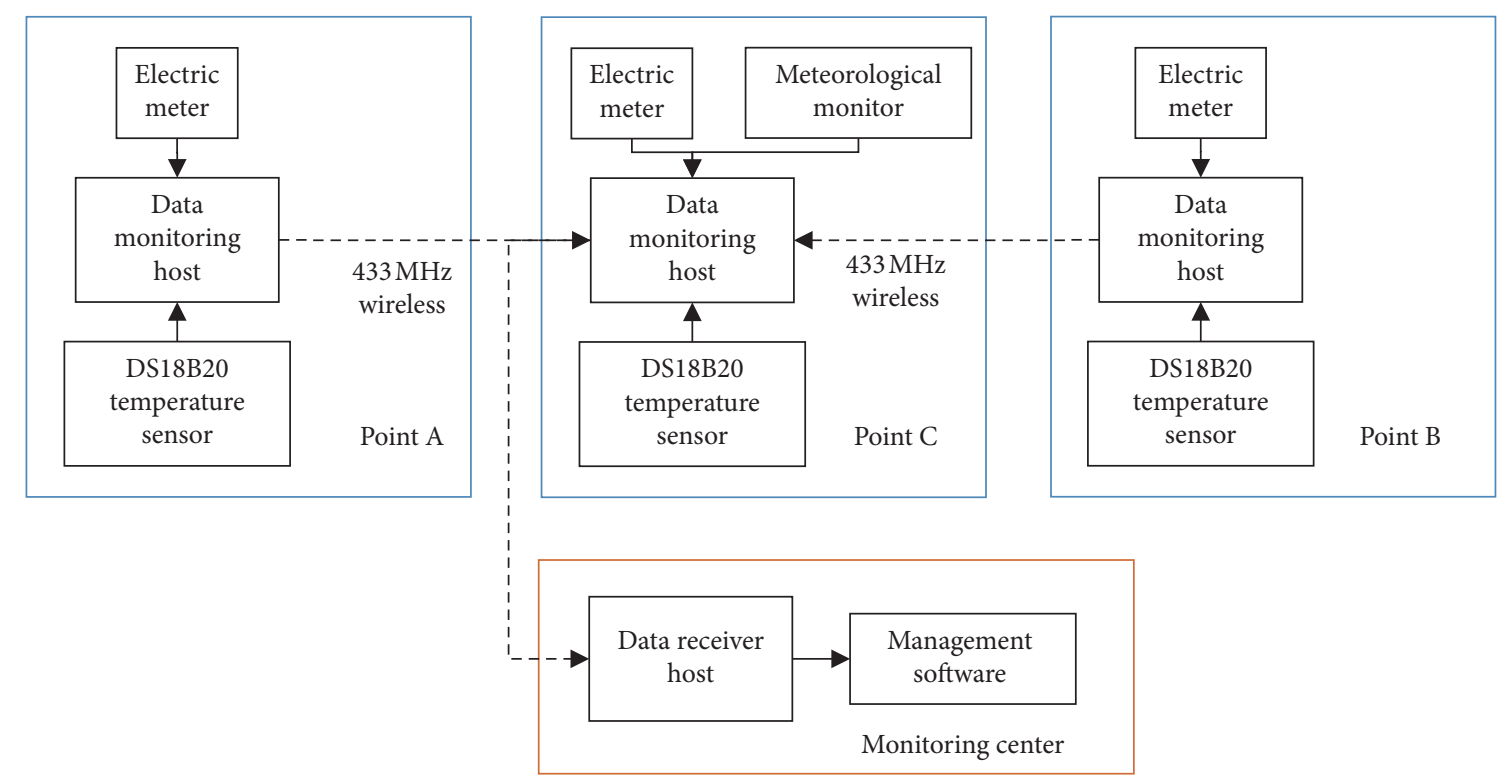

FIgURE 1: The structure of the MSIF monitoring system.

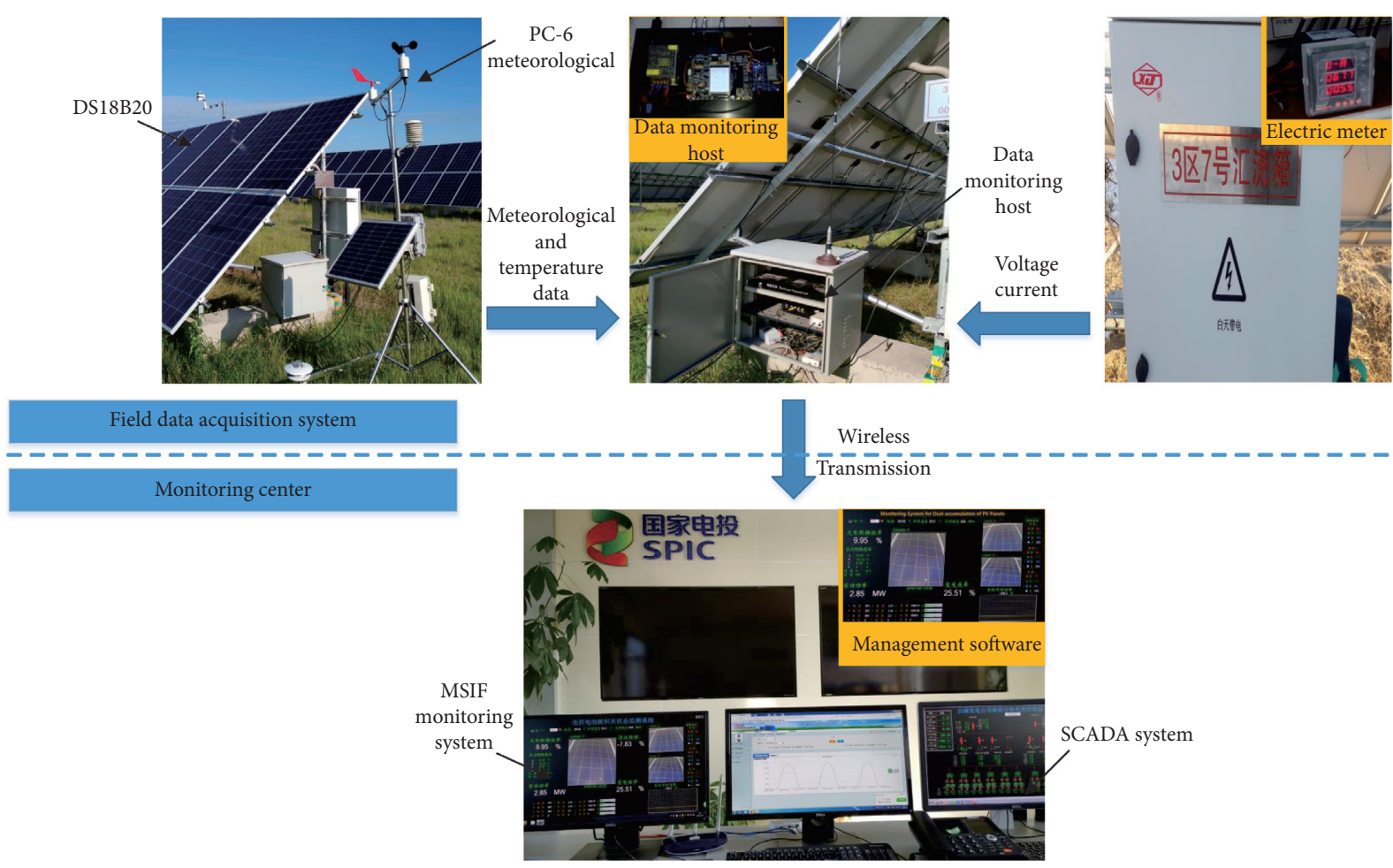

Figure 2: The MSIF monitoring system at the experimental site.

shown in Figure 3, take the time series with an interval of 15 minutes and the rolling time sample data set is established. Through the observation of the curve, it can be concluded that the temperature change of PV panels is caused by a variety of factors and has the characteristics of being random and nonlinear.

\section{Methodology}

In this section, the principle of SVM will be given in subsection 3.1. Three optimization methods, PSO algorithm, DE algorithm, and PIO algorithm, will be briefly described in subsections 3.2, 3.3, and 3.4. 


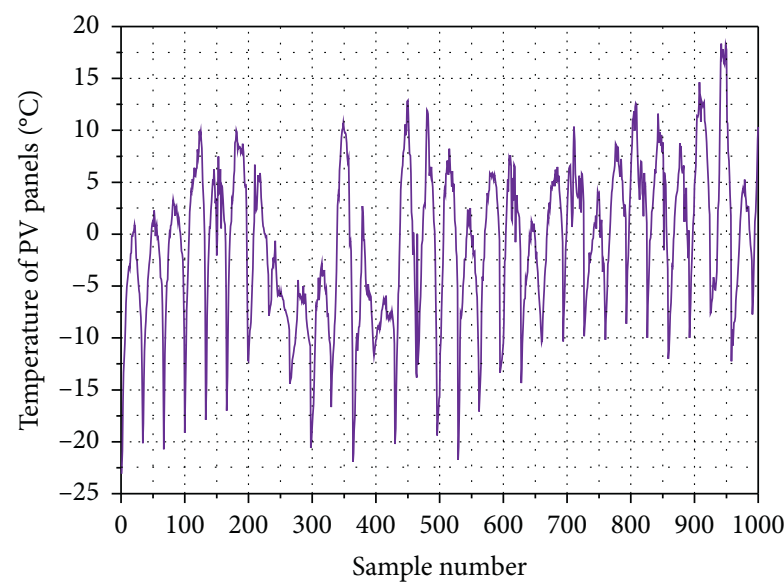

Figure 3: The recorded temperature data of PV panels.

3.1. SVM Model. SVM is a data-based machine learning method, which adopts the principle of risk minimization. It has good generalization performance and has obvious advantages in solving small sample problems. In the process of establishing the SVM model, the training network is firstly trained with the training sample data, and then the network is used for later prediction. The training sample set $\left\{\left(x_{i}, y_{i}\right) \mid i t=n 1,2,3 q, h \ldots, x n\right\}$, where $x_{i} \in R^{n}, y_{i} \in R$. The function of SVM is described as follows:

$$
f(x)=w^{T} \cdot x_{i}+b,
$$

where $w^{T}$ denotes weight and $b$ denotes offset value. In order to find the optimal parameters $w$ and $b$, the function is constructed:

$$
\begin{array}{ll}
\min & \frac{1}{2} w^{T} w+c \sum_{i=1}^{n} \zeta_{i} \\
\text { s.t. } & y_{i}\left(w x_{i}+b_{i}\right) \geq 1-\zeta_{i} \\
& \zeta_{i} \geq 0, \quad i=1,2,3, \ldots, l,
\end{array}
$$

where parameter $c$ denotes penalty factor and $\zeta$ denotes relaxation variables. Combined with the optimization principle, equation (3) is transformed as follows:

$$
\begin{aligned}
& \min \frac{1}{2} \sum_{i, j=1}^{l}\left(a_{i}^{*}-a_{i}\right)\left(a_{j}^{*}-a_{j}\right) k\left(x_{i}, x_{j}\right)+\varepsilon \sum_{i=1}^{l}\left(a_{i}^{*}+a_{i}\right)-\sum_{i=1}^{l} y_{i}\left(a_{i}^{*}-a_{i}\right) \\
& \text { s.t. } \quad \sum_{i}^{l}\left(a_{i}^{*}-a_{i}\right)=0 \\
& a_{i}^{*} \geq 0, a_{i} \geq \frac{c}{l}, \quad i=1,2,3, \ldots, l,
\end{aligned}
$$

where $k(x i, x j)$ is the kernel function of SVM and $a_{i}^{*}, a_{i}$ are the Lagrange multiplier. The RBF kernel function is selected, and its expression is as follows:

$$
k\left(x_{i}, x_{j}\right)=\exp \left(-g\left\|x-x_{i}\right\|^{2}\right), \quad g>0,
$$

where $g$ is a parameter of the kernel function.

3.2. PSO Algorithm. PSO is a kind of evolutionary computation, which originated from the research on the hunting behavior of birds. It has been widely used in function optimization, neural network training, fuzzy system control, and other applications. The velocity and position of a particle are two important properties, which search for the optimal solution in search space. Firstly, all the particles in space are assigned initial random positions and initial random velocities. Secondly, the individual optimal solution for each particle is calculated. Then, the individual particle can share extreme value information with other particles in the particle swarm. Finally, the optimal solution is sought through the interaction and information transfer between particles.

3.3. DE Algorithm. DE is developed from the genetic algorithm (GA), an algorithm model that simulates biological evolution. Through the operation of the difference operator, the population is continuously evolved and iterated to save the individual with the highest adaptability, which is often used to find the optimal solution of nonlinear and nondifferentiable problems, and it has the characteristics of fast speed and good robustness. DE algorithm approaches to the global optimal solution through repeated iteration on the population, which mainly includes population initialization, mutation operation, crossover operation, and selection operation.

3.4. PIO Algorithm. PIO is a novel intelligence optimization algorithm for solving global optimization problems, and it is to simulate the homing behavior of pigeon by the Earth's magnetic field and landmarks [42]. When pigeon is far away from its destination, it uses the Earth's magnetic to identify the direction. When pigeon is close to its destination, it uses landmarks for navigation.

3.4.1. Map and Compass Operator. In the map and compass operator, the rules are defined with the position $X_{i}$ and the velocity $V_{i}$ of $i$ pigeon, and the positions and velocities in a D-dimension search space are updated in each iteration. The new position $X_{i}$ and velocity $V_{i}$ of $i$ pigeon at the th iteration can be calculated as follows: 


$$
\begin{aligned}
& V_{i}(n)=V_{i}(n-1) * e^{-R n}+\operatorname{rand} *\left(X_{\text {gbest }}-X_{i}(n-1)\right), \\
& X_{i}(n)=X_{i}(n-1)+V_{i}(n),
\end{aligned}
$$

where $R$ denotes map and compass factor, rand denotes a random number, and $X_{\text {gbest }}$ denotes the current global best position.

3.4.2. Landmark Operator. In landmark operator, $N_{p}$ is used to record the half number of pigeons in each generation, and $X_{c}(n)$ is the center position of all pigeons in generation $n$. If each pigeon can fly a direct distance to the destination, the position updating rule for pigeon $i-$ th iteration is described as follows:

$$
\begin{gathered}
N_{p}(n)=\frac{N_{p}(n-1)}{2}, \\
X_{c}(n)=\frac{\sum X_{i}(n) * \text { fitness }\left(X_{i}(n)\right)}{N_{p} * \sum \text { fitness }\left(X_{i}(n)\right)}, \\
X_{i}(n)=X_{i}(n-1)+\operatorname{rand} *\left(X_{c}(n)-X_{i}(n-1)\right),
\end{gathered}
$$

where fitness $(x)$ denotes the quality of the pigeon individual. For the minimum optimization problems, fitness $\left(X_{i}(n)\right)=1 /\left(f_{\min }\left(X_{i}(n)\right)+\varepsilon\right)$. On the contrary, fitness $\left(X_{i}(n)\right)=f_{\max }\left(X_{i}(n)\right)$.

\section{DFPIO-SVM}

The SVM for regression accuracy depends on the parameters of penalty factor $c$ and kernel function $g$. The selection of these two parameters is generally obtained through experience and tends to affect the effect of regression. In order to improve the regression accuracy of SVM, DFPIO will be used to optimize the selection of these parameters.

4.1. DFPIO. During the updating iteration of pigeon, the direction of movement of the pigeon is always to the optimal position. The velocity change of pigeon $i$ at time $t$ is

$$
\Delta V_{i}(t+1)=V_{i}(t+1)-V_{i}(t) .
$$

When $\Delta V_{i}(t+1) \Delta V_{i}(t)<0$, it shows that the pigeon is in reverse motion at $t+1$ and $t$ moment, and the pigeon velocity is too large to pass the current optimal solution at $t$ moment. At this time, the flying velocity of pigeon should be reduced to make it close to the optimal solution. In order to improve the accuracy of the pigeon, we refer to the delay factor (DF) $\tau$ into the position update equation (8). So, the delay factor $\tau$ is given by

$$
\begin{aligned}
& \tau=\tau_{\max }-\frac{\left(\tau_{\max }-\tau_{\min }\right)}{N_{i t e r}} * i, \\
& \tau \in[0.9,1] .
\end{aligned}
$$

According to the changing characteristics of the velocity before and after the pigeon, DF can dynamically adjust the flying speed of the pigeon. Thus, the position update can be rewritten as follows:

$$
X_{i}(n)=X_{i}(n-1)+\tau V_{i}(n) .
$$

4.2. DFPIO-SVM. DFPIO algorithm is used to optimize the penalty factor $c$ and kernel function $g$ of SVM. The parameters are treated as two particles, which constantly update their positions and velocities, and their fitness values are calculated by the objective function to achieve the global optimum.

An algorithmic implementation for the optimization process is provided in Algorithm 1.

\section{Results}

In this section, the Mackey-Glass chaotic time series and Nonlinear Autoregressive Moving-Average (NARMA) series are used to verify the performance of DFPIO-SVM. DFPIO-SVM is compared to original SVM [20], PSO [29], DE [38], PIO [42], and OPIO [48]. For these prediction methods, the training number of samples is 900 , and the prediction number of samples is 100 . The dimension of the input and output are 5 and 1, respectively. For optimization methods, the size of the swarm optimization algorithm $n$ is 30 , particle vector dimension is 2 , the map and compass factor $R$ is 0.3 , and the number of iterations $N_{\text {iter }}$ is 30 .

5.1. The Mackey-Glass Chaotic Time Series. The Mackey-Glass is a typical chaotic system, which is selected to verify the prediction performance of DFPIO-SVM. It can be described by

$$
y(n)=y(n-1)+0.1\left(\frac{0.2 y(n-10 \tau)}{1+y(n-10 \tau)^{10}}-0.1 y(n-1)\right),
$$

where $\tau$ is set as 17 . The Runge-Kutta method is used to generate the Mackey-Glass time series and the initial condition $(y(0)=1.2)$. The mean squared errors (MSE) and root mean squared error (RMSE) are given to analyse the performance of prediction methods, which can be described by

$$
\begin{aligned}
\text { MSE } & =\frac{1}{N} \sum_{i=1}^{N}\left(y_{n}-\hat{y}_{n}\right)^{2}, \\
\text { RMSE } & =\sqrt{\frac{1}{N} \sum_{i=1}^{N}\left(y_{n}-\hat{y}_{n}\right)^{2},}
\end{aligned}
$$

where $y_{n}$ denotes target data, $\hat{y}_{n}$ denotes predicted data, and $N$ is the number of data samples.

The prediction accuracy of DFPIO-SVM and other methods in $[20,29,38,42,48]$ are shown in Table 1, and it 
Input: number of pigeons $n$, number of iterations for optimization $N_{\text {iter }}$, the map and compass factor $R$; Output: trained DFPIO-SVM;

(1) Step 1: initialize $N_{\text {iter }}$ and $n$ pigeons positions at random $\in[0.01,20]$;

(2) Step 2: compute the fitness of each pigeon;

(3) Step 3: update velocity $V_{i}$ and position $X_{i}$ according to equations (7) and (15);

(4) Step 4: update position $X_{i}$ according to equation (11);

(5) Step 5: update $c$ and $g$;

(6) Step 6: obtain optimal MSE.

Algorithm 1: DFPIO-SVM algorithm.

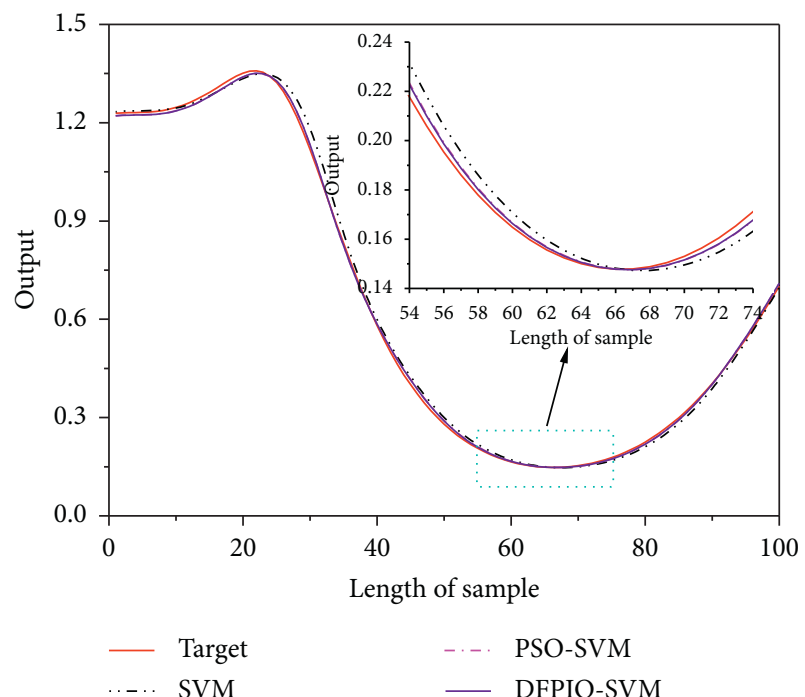

FIGURE 4: The target and prediction output for the Mackey-Glass time series.

can be seen that the prediction accuracy of the DFPIO-SVM

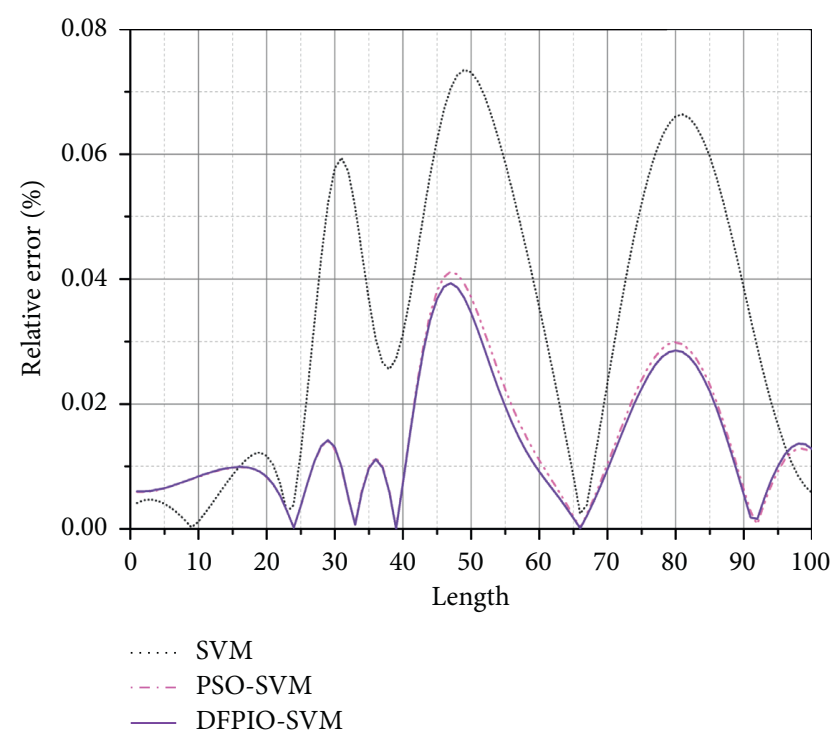

Figure 5: The prediction error curves of three methods for the Mackey-Glass time series. is better than that of those methods. The average prediction accuracies of DFPIO-SVM are increased by $84.08 \%$.

For the Mackey-Glass chaotic time series, the prediction output values of five methods and the target values are shown in Figure 4. From this figure, we can see that the DFPIO-SVM fits the curve moderately well. Figure 5 shows the prediction error of three methods. The prediction error curves of the five methods fluctuate, but the curve of DFPIOSVM is the lowest. The average prediction errors of SVM, PSO-SVM, and DFPIO-SVM are 0.03306, 0.01434, and 0.01373 , respectively.

The trained SVM model is used to predict the Mackey-Glass chaotic time series. Comparing the fitness results of MSE shown in Figure 6, it can be seen that the DFPIO algorithm achieves the smallest MSE within $23^{\text {rd }}$ iterations. PIO algorithm achieves the same MSE as DFPIO at the $15^{\text {th }}$ iteration. In the iteration process, the fitness values of PSO are higher than PIO and DFPIO.

5.2. NARMA Series. At the same time, a NARMA model is given to verify the prediction performance of DFPIO-SVM, which can be described as follows:

$$
\begin{aligned}
y(n)= & 0.3 y(n-1)+0.05 y(n-1)\left(\sum_{i=1}^{10} y(n-i)\right) \\
& +1.5 u(n-10) u(n-1)+0.1,
\end{aligned}
$$

where $y(n)$ and $u(n)$ are output and input. The input $u(n)$ is independent identically distributed random samples, and its value is in the range $[0,1]$. The output $y(n)$ is the value initialized in the range $[-1,1]$.

For the NARMA series, the prediction accuracies of the five methods are shown in Table 2. It is apparent from this table that DFPIO-SVM can be compared with other methods, having better prediction performance. The average prediction accuracy of DFPIO-SVM increased by $2.31 \%$.

Figure 7 shows the target values and prediction output values of three methods, and the corresponding error curve is shown in Figure 8. The prediction results of three methods fluctuated greatly, and the prediction of peak value is not accurate. The overall trend of the DFPIO-SVM model is close to the actual prediction data, compared with the other two prediction methods, it has a better fitting degree with the actual data. The average prediction errors of DE-SVM, 


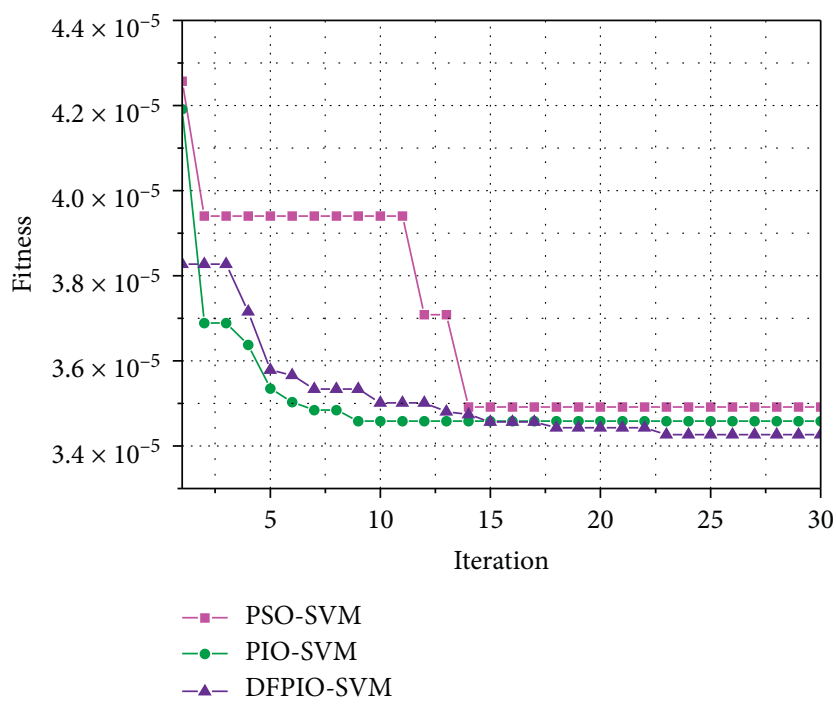

FIgURE 6: Iterative curves of three optimization methods.

TABLE 1: Comparison of prediction accuracy for the Mackey-Glass time series.

\begin{tabular}{|c|c|c|c|c|c|}
\hline \multirow{2}{*}{ Method } & \multicolumn{2}{|c|}{ Optimized parameters } & \multirow{2}{*}{ MSE } & \multirow{2}{*}{ RMSE } & \multirow{2}{*}{ Accuracy improvement (\%) } \\
\hline & $c$ & $g$ & & & \\
\hline SVM & 0.1 & 1 & $4.1113 e-04$ & $2.03 e-02$ & - \\
\hline DE-SVM & 17.7584 & 1.5187 & $1.2462 e-04$ & $1.12 e-02$ & 69.69 \\
\hline PSO-SVM & 16.2988 & 0.6093 & $6.6701 e-05$ & $8.18 e-03$ & 83.78 \\
\hline PIO-SVM & 18.6471 & 0.6140 & $6.6069 e-05$ & $8.13 e-03$ & 83.90 \\
\hline OPIO-SVM & 16.8580 & 0.6129 & $6.6346 e-05$ & $8.14 e-03$ & 83.86 \\
\hline DFPIO-SVM & 16.7831 & 0.6151 & $6.5455 e-05$ & $8.09 e-03$ & 84.08 \\
\hline
\end{tabular}

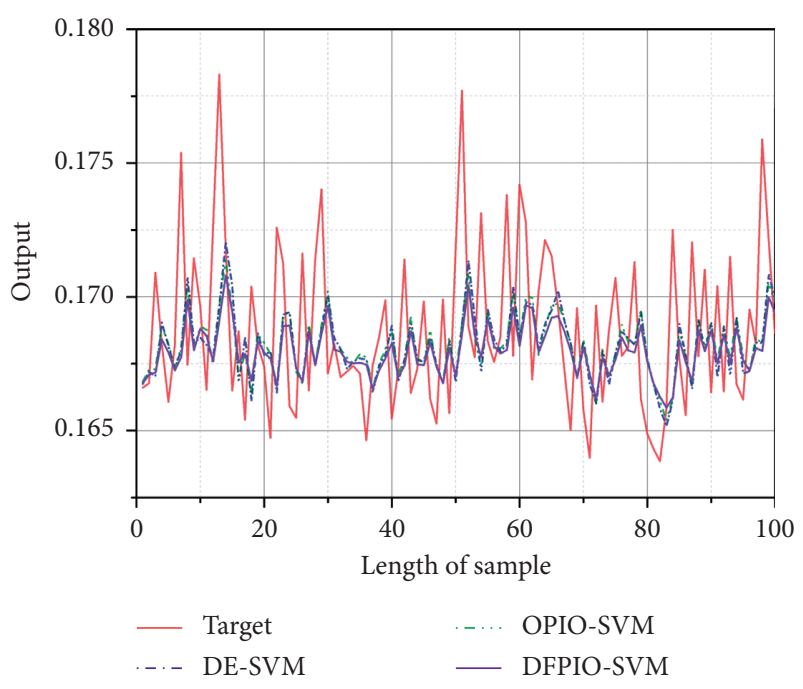

Figure 7: The target and prediction output for NARMA.

OPIO-SVM, and DFPIO-SVM are 0.01537, 0.01516, and 0.01466 , respectively.

In Figure 9, we can see that the DFPIO algorithm achieves the smallest MSE within the $25^{\text {th }}$ iterations. PIO algorithm achieves the same MSE as PSO at the $15^{\text {th }}$ iteration. With the increase in iteration, it can be seen that the delay factor increases the global search capability of the PIO algorithm.

5.3. The Temperature of $P V$ Panels. To further verify the prediction performance of DFPIO-SVM for the temperature of PV panels, the field temperature data collected in PV power station from Jilin are tested in this paper. The mean absolute percentage error (MAPE) and its expression are as follows:

$$
\text { MAPE }=\frac{1}{N} \sum_{i=1}^{N}\left|\frac{y_{n}-\hat{y}_{n}}{y_{n}}\right| \times 100 \%,
$$

where $y_{n}$ denotes target data, $\hat{y}_{n}$ denotes predicted data, and $N$ is the number of data samples.

As can be seen from Table 3, DFPIO-SVM achieved the smallest MAPE value. After executing the DFPIO algorithm, search and get the optimal SVM parameter $c=10.2261$, $g=0.6090$. It can be intuitively seen that the established prediction algorithm is more effective and less affected by sample data. The DFPIO-SVM algorithm can be used to find the optimal parameters of SVM more easily and efficiently, which improves the prediction accuracy of the model.

The best parameters of five optimization methods are brought into the SVM model for PV panels temperature prediction. The predicted results are shown in Figure 10, and 


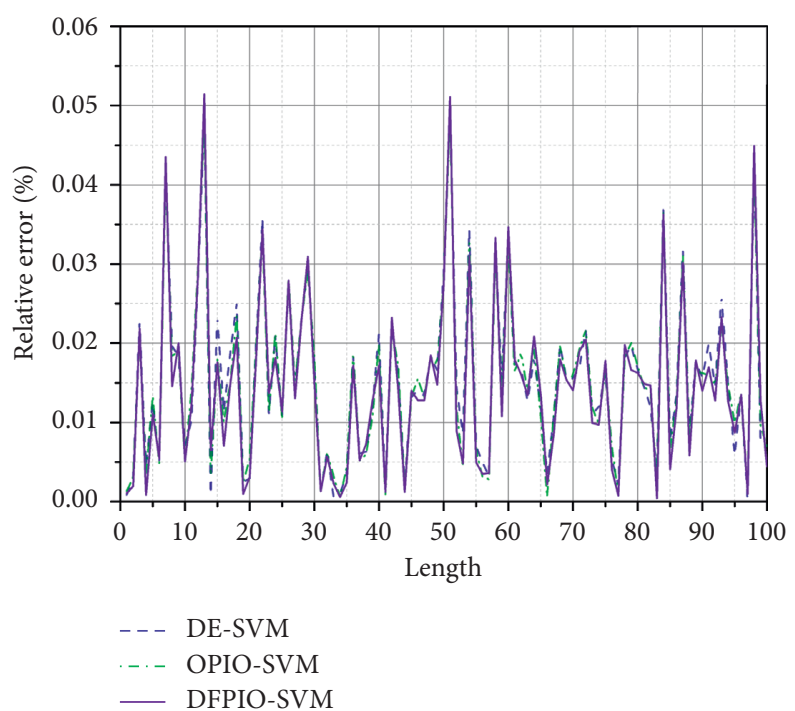

FIgURE 8: The prediction error curves of three methods for NARMA.

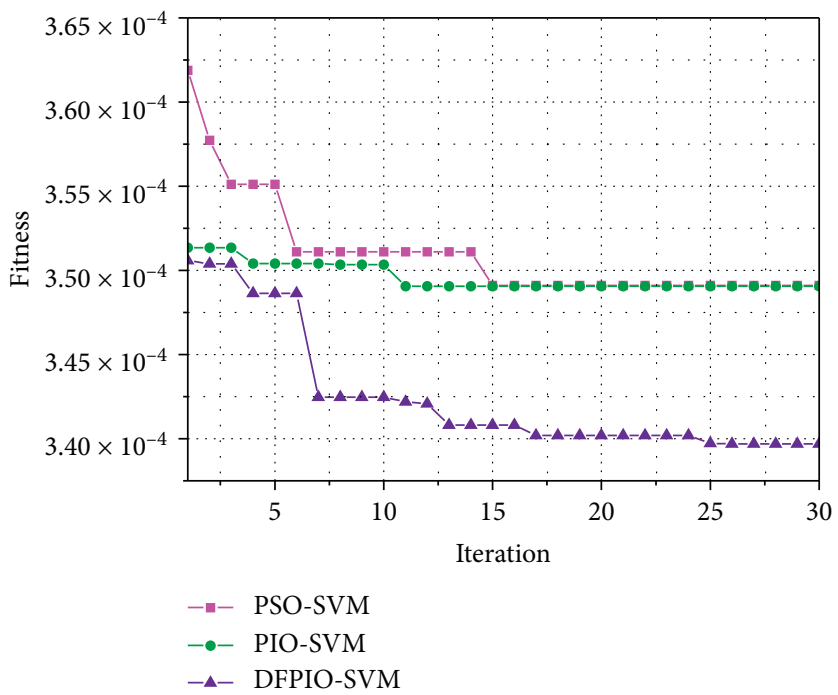

FIGURE 9: Iterative curves of three optimization methods.

TABLE 2: Comparison of prediction accuracy for NARMA.

\begin{tabular}{|c|c|c|c|c|c|}
\hline \multirow{2}{*}{ Method } & \multicolumn{2}{|c|}{ Optimized parameters } & \multirow{2}{*}{ MSE } & \multirow{2}{*}{ RMSE } & \multirow{2}{*}{ Accuracy improvement (\%) } \\
\hline & $c$ & $g$ & & & \\
\hline SVM & 0.1 & 1 & $1.0155 e-05$ & $3.187 e-03$ & - \\
\hline DE-SVM & 1.8581 & 13.3556 & $1.0340 e-05$ & $3.216 e-03$ & -1.82 \\
\hline PSO-SVM & 0.0719 & 7.9756 & $1.0141 e-05$ & $3.184 e-03$ & 0.13 \\
\hline PIO-SVM & 0.6372 & 12.5727 & $1.0140 e-05$ & $3.184 e-03$ & 0.14 \\
\hline OPIO-SVM & 0.0772 & 13.4447 & $1.0075 e-05$ & $3.174 e-03$ & 0.79 \\
\hline DFPIO-SVM & 0.0130 & 10.0047 & $9.9202 \mathrm{e}-06$ & $3.150 \mathrm{e}-03$ & 2.31 \\
\hline
\end{tabular}




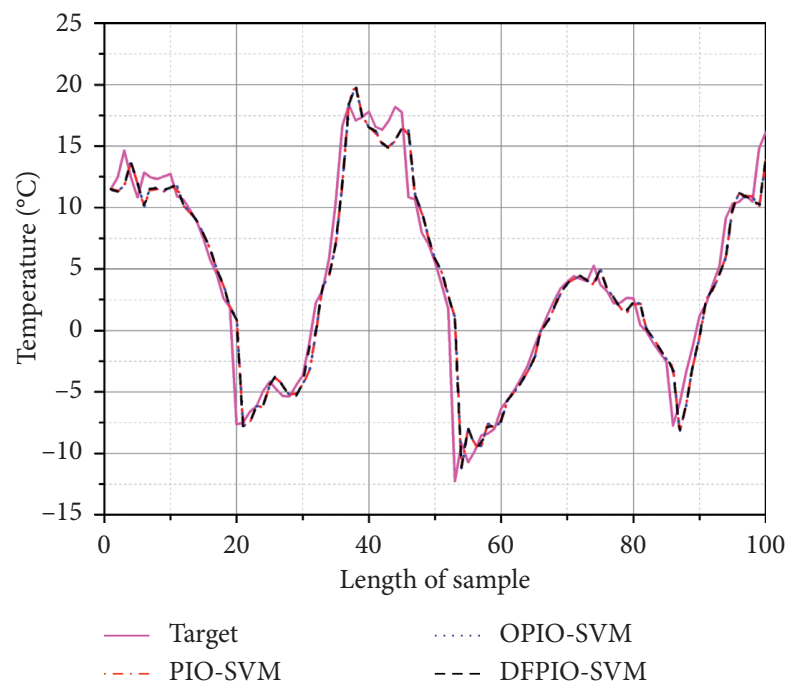

FIgURE 10: The target and prediction output for the temperature of PV panels.

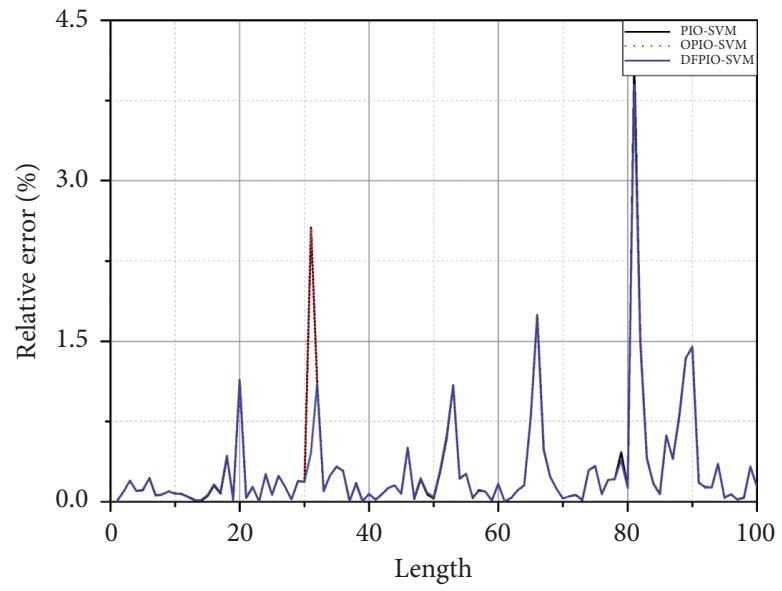

FIgURE 11: The prediction error curves of three methods for the temperature of PV panels.

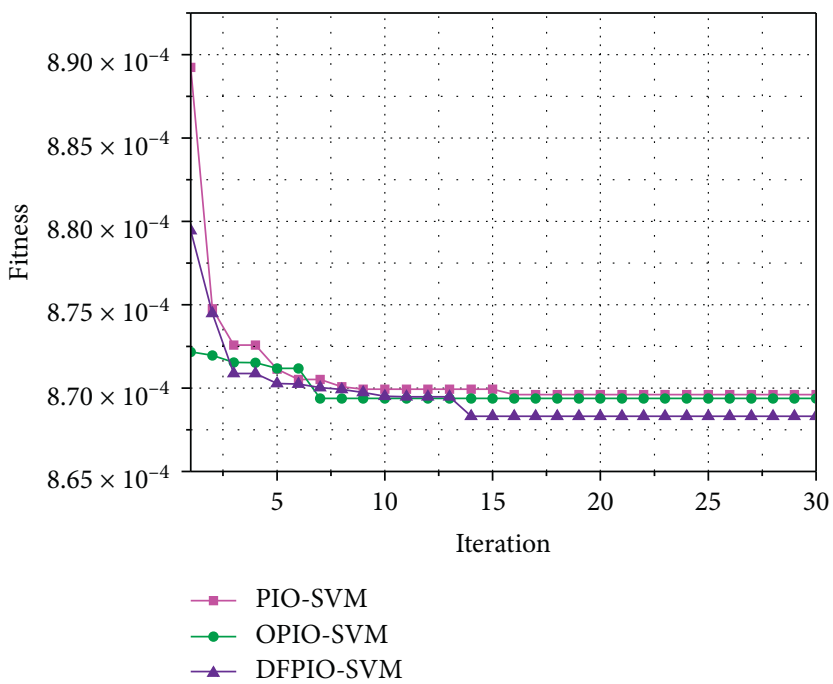

FIgURE 12: Iterative curves of three optimization methods. 
TABLE 3: Comparison of prediction accuracy for the temperature of PV panels.

\begin{tabular}{lccc}
\hline Method & MAPE (\%) & Accuracy improvement (\%) & Training time (s) \\
\hline SVM & 0.4172 & - & - \\
DE-SVM & 0.3130 & 28.08 & - \\
PSO-SVM & 0.3138 & 27.91 & 128.595743 \\
PIO-SVM & 0.3121 & 28.51 & 116.0571 \\
OPIO-SVM & 0.3104 & 28.59 & 103.87497 \\
DFPIO-SVM & $\mathbf{0 . 2 8 8 3}$ & 28.97 & 94.505892 \\
\hline
\end{tabular}

the prediction relative error is shown in Figure 11. According to the running results of the program, the prediction results based on DFPIO-SVM are in better agreement with the target. The average percentage error of the test prediction is $0.2883 \%$. The simulation results show that the DFPIO-SVM can achieve high accuracy in the temperature prediction of PV panels.

A temperature data set of PV panels is used for SVM training in Figure 12. The results show that DFPIO-SVM converges faster than other algorithms. DFPIO-SVM algorithm achieves the smallest MSE at the $14^{\text {th }}$ iteration, and it can achieve the least iterations.

\section{Conclusions}

In this paper, a novel DFPIO algorithm is proposed, which is used to solve the problem of SVM parameters optimization. By referring the delay factor to the position updating equation, the convergence accuracy of the PIO algorithm is improved. Meanwhile, the predictive performance of the proposed algorithm is verified by the Mackey-Glass chaotic time series and NARMA series. In order to further analyse the application effect of the proposed model in the prediction temperature of PV panels, a MSIF monitoring system is established in a PV power station from Jilin. The temperature data of PV panels, from January to June 2018, are selected for the input of the prediction model. Comparing with other optimization methods, the effectiveness of the DFPIO algorithm is verified. These results show that the DFPIO-SVM model can be used to better predict the temperature of PV panels and have strong generalization ability.

\section{Data Availability}

The MATLAB data used to support the findings of this study are available from the corresponding author upon request.

\section{Conflicts of Interest}

The authors declare that there are no conflicts of interest regarding this paper.

\section{Acknowledgments}

This work was supported by the National Key R \& D Program of China (Grant no. 2018YFB1500800), Science and Technology Development Program of Jilin Province (Grant no. 20190302079GX), and Science and Technology
Innovation Development Program of Jilin City (Grant no. 201830819).

\section{References}

[1] A. Al-Dousari, W. Al-Nassar, A. Al-Hemoud et al., "Solar and wind energy: challenges and solutions in desert regions," Energy, vol. 176, pp. 184-194, 2019.

[2] L. Micheli, J. A. Caballero, E. F. Fernandez et al., "Correlating photovoltaic soiling losses to waveband and single-value transmittance measurements," Energy, vol. 180, pp. 376-386, 2019.

[3] M. Bashir, H. Ali, M. Ali, and A. Siddiqui, “An experimental investigation of performance of photovoltaic modules in Pakistan," Thermal Science, vol. 19, no. 2, pp. 525-534, 2015.

[4] H. Ali, M. Mahmood, M. A. Bashir et al., "Outdoor testing of photovoltaic modules during summer in Taxila, Pakistan," Thermal Science, vol. 20, pp. 165-173, 2014.

[5] M. M. Rahman, M. Hasanuzzaman, and N. A. Rahim, "Effects of operational conditions on the energy efficiency of photovoltaic modules operating in Malaysia," Journal of Cleaner Production, vol. 143, pp. 912-924, 2017.

[6] L. Xu, S. Li, J. Jiang et al., "The influence of dust deposition on the temperature of soiling photovoltaic glass under lighting and windy conditions," Solar Energy, vol. 199, pp. 491-496, 2020.

[7] W. C. L. Kamuyu, J. Lim, C. Won et al., "Prediction model of photovoltaic module temperature for power performance of floating PVs," Energies, vol. 11, p. 447, 2018.

[8] H. Qu and J. Wang, "Theoretical and experimental study on the influence of ash deposition on photovoltaic panel output characteristics," Solar Energy, vol. 39, pp. 2335-2340, 2018.

[9] C. Wang, A. Yang, J. Li et al., "Experimental study on the influence of multi-factor coupling on the surface temperature of photovoltaic modules," Solar Energy, vol. 40, pp. 112-118, 2019.

[10] A. Dhaundiyal and D. Atsu, "The effect of wind on the temperature distribution of photovoltaic modules," Solar Energy, vol. 201, pp. 259-267, 2020.

[11] M. Almaktar, H. A. Rahman, M. Y. Hassan, and S. Rahman, "Climate-based empirical model for PV module temperature estimation in tropical environment," Applied Solar Energy, vol. 49, no. 4, pp. 192-201, 2013.

[12] E. Barykina and A. Hammer, "Modeling of photovoltaic module temperature using Faiman model: sensitivity analysis for different climates," Solar Energy, vol. 146, pp. 401-416, 2017.

[13] A. M. Muzathik, "Photovoltaic modules operating temperature estimation using a simple correlation," International Journal of Energy Engineering, vol. 4, pp. 151-158, 2014.

[14] I. Ceylan, O. Erkaymaz, E. Gedik, and A. E. Gürel, "The prediction of photovoltaic module temperature with artificial 
neural networks," Case Studies in Thermal Engineering, vol. 3, pp. 11-20, 2014.

[15] B. Wang, L. Zhang, H. Ma, H. Wang, and S. Wan, "Parallel LSTM-based regional integrated energy system multienergy source-load information interactive energy prediction," Complexity, vol. 2019, Article ID 7414318, 13 pages, 2019.

[16] H. Zheng, J. J. Peng, H. J. Lian et al., "Deep recurrent model for server load and performance prediction in data center," Complexity, vol. 2017, Article ID 8584252, 10 pages, 2017.

[17] Y. Wu, Y. Ding, Y. Zhu, J. Feng, and S. Wang, "Complexity to forecast flood: problem definition and spatiotemporal attention LSTM solution," Complexity, vol. 2020, Article ID 7670382, 13 pages, 2020.

[18] Z. Yu, L. Qin, Y. Chen, and M. D. Parmar, "Stock price forecasting based on LLE-BP neural network model," Physica A: Statistical Mechanics and Its Applications, vol. 553, Article ID 124197, 2020.

[19] F. Liu, H. Gong, L. Cai et al., "Prediction of ammunition storage reliability based on improved ant colony algorithm and bp neural network," Complexity, vol. 2019, Article ID 5039097, 2019.

[20] G. N. Kouziokas, "A new W-SVM kernel combining PSOneural network transformed vector and Bayesian optimized SVM in GDP forecasting," Engineering Applications of Artificial Intelligence, vol. 92, Article ID 103650, 2020.

[21] M. Alfaro, G. Fuertes, M. Vargas et al., "Forecast of chaotic series in a horizon superior to the inverse of the maximum lyapunov exponent," Complexity, vol. 2018, Article ID 1452683, 9 pages, 2018.

[22] Z. Yang, M. Mourshed, K. Liu et al., "A novel competitive swarm optimized RBF neural network model for short-term solar power generation forecasting," Neurocomputing, vol. 397, 2020.

[23] M.-w. Shi, "Based on time series and RBF network plant disease forecasting," Procedia Engineering, vol. 15, pp. 23842387, 2011.

[24] R. Mohammadi, S. M. T. Fatemi Ghomi, and F. Zeinali, "A new hybrid evolutionary based RBF networks method for forecasting time series: a case study of forecasting emergency supply demand time series," Engineering Applications of Artificial Intelligence, vol. 36, pp. 204-214, 2014.

[25] X. Yao, Z. Wang, and H. Zhang, "Identification method for a class of periodic discrete-time dynamic nonlinear systems based on Sinusoidal ESN," Neurocomputing, vol. 275, pp. 1511-1521, 2018.

[26] Q. Zhang, H. Qian, Y. Chen et al., "A short-term traffic forecasting model based on echo state network optimized by improved fruit fly optimization algorithm," Neurocomputing, vol. 416, 2019.

[27] X. Yao, Z. Wang, and H. Zhang, "Prediction and identification of discrete-time dynamic nonlinear systems based on adaptive Echo state network," Neural Networks, vol. 113, pp. 11-19, 2019.

[28] Z. Tang and Z. Zhang, "The multi-objective optimization of combustion system operations based on deep data-driven models," Energy, vol. 182, pp. 37-47, 2019.

[29] A. Selakov, D. Cvijetinović, L. Milović, S. Mellon, and D. Bekut, "Hybrid PSO-SVM method for short-term load forecasting during periods with significant temperature variations in city of Burbank," Applied Soft Computing, vol. 16, pp. 80-88, 2014.

[30] A. Zhang, P. Zhang, and Y. Feng, "Short-term load forecasting for microgrids based on DA-SVM," COMPEL - The International Journal for Computation and Mathematics in
Electrical and Electronic Engineering, vol. 38, no. 1, pp. 68-80, 2019.

[31] W. Ma, X. Zhang, Y. Xin, and S. Li, "Study on short-term network forecasting based on SVM-MFA algorithm," Journal of Visual Communication and Image Representation, vol. 65, p. 102646, 2019.

[32] J. Lu, X. Liao, S. Li et al., "An effective ABC-SVM approach for surface roughness prediction in manufacturing processes," Complexity, vol. 8, pp. 1-13, 2019.

[33] P. J. García Nieto, J. R. Alonso Fernández, E. García-Gonzalo, C. Díaz Muñiz, R. M. Bayón, and V. M. González Suárez, "A new predictive model for the cyanotoxin content from experimental cyanobacteria concentrations in a reservoir based on the ABC optimized support vector machine approach: a case study in Northern Spain," Ecological Informatics, vol. 30, pp. 49-59, 2015.

[34] A. S. Oshaba, E. S. Ali, and M. A. Sahar, "PI controller design using artificial bee colony algorithm for MPPT of photovoltaic system supplied DC motor-pump load," Complexity, vol. 21, no. 6, pp. 99-111, 2016.

[35] L. Chen, Y. Feng, R. Li, X. Chen, and H. Jiang, "Jiles-atherton based hysteresis identification of shape memory alloy-actuating compliant mechanism via modified particle swarm optimization algorithm," Complexity, vol. 2019, Article ID 7465461, 11 pages, 2019.

[36] G. N. Kouziokas, "SVM kernel based on particle swarm optimized vector and Bayesian optimized SVM in atmospheric particulate matter forecasting," Applied Soft Computing, vol. 93, p. 106410, 2020.

[37] W. Yan, M. Li, X. Pan, G. Wu, and L. Liu, "Application of support vector regression cooperated with modified artificial fish swarm algorithm for wind tunnel performance prediction of automotive radiators," Applied Thermal Engineering, vol. 164, p. 114543, 2020.

[38] P. Millie, Z. Hira, L. Garcia-Hernandez, and A. Ajith, "Differential evolution: A review of more than two decades of research," Engineering Applications of Artificial Intelligence, vol. 90, Article ID 103479, 2020.

[39] Y. Tang, J. Ji, Y. Zhu et al., "A differential evolution-oriented pruning neural network model for bankruptcy prediction," Complexity, vol. 2019, Article ID 8682124, 21 pages, 2019.

[40] Y. Wang, Z. Yang, M. Mourshed, Y. Guo, Q. Niu, and X. Zhu, "Demand side management of plug-in electric vehicles and coordinated unit commitment: a novel parallel competitive swarm optimization method," Energy Conversion and Management, vol. 196, pp. 935-949, 2019.

[41] Z. Yang, K. Li, Y. Guo et al., "A binary symmetric based hybrid meta-heuristic method for solving mixed integer unit commitment problem integrating with significant plug-in electric vehicles," Energy, vol. 170, pp. 889-905, 2019.

[42] H. Duan and P. Qiao, "Pigeon-inspired optimization: a new swarm intelligence optimizer for air robot path planning," International Journal of Intelligent Computing and Cybernetics, vol. 7, no. 1, pp. 24-37, 2014.

[43] H. B. Duan, H. X. Qiu, and Y. M. Fan, "Optimal combat coordination control for unmanned aerial vehicle using predator-escaped pigeon," Chinese Science: Technological Sciences, vol. 45, pp. 559-572, 2015.

[44] X. Zhang, H. B. Duan, and C. Yang, "Pigeon-inspired optimization approach to multiple UAVs formation reconfiguration controller design," in Proceedings of 2014 IEEE Chinese Guidance, Navigation and Control Conference, pp. 2707-2712, Yantai, China, August 2014. 
[45] Y. Deng and H. Duan, "Control parameter design for automatic carrier landing system via pigeon-inspired optimization," Nonlinear Dynamics, vol. 85, no. 1, pp. 97-106, 2016.

[46] S. Zhang and H. Duan, "Gaussian pigeon-inspired optimization approach to orbital spacecraft formation reconfiguration," Chinese Journal of Aeronautics, vol. 28, no. 1, pp. 200-205, 2015.

[47] D. Zhang, H. Duan, Y. Yang et al., "Active disturbance rejection control for small unmanned helicopters via levy flightbased pigeon-inspired optimization," Aircraft Engineering and Aerospace Technology, vol. 89, no. 6, pp. 946-952, 2017.

[48] H. Duan and X. Wang, "Echo state networks with orthogonal pigeon-inspired optimization for image restoration," IEEE Transactions on Neural Networks and Learning Systems, vol. 27, no. 11, pp. 2413-2425, 2016.

[49] X. Lei, Y. Ding, and F. X. Wu, "Detecting protein complexes from DPINs by density based clustering with Pigeon-Inspired Optimization Algorithm," Science China Information Sciences, vol. 59, pp. 1-14, 2016.

[50] Z. Yang, K. Liu, J. Fan et al., "A novel binary/real-valued pigeon inspired optimization for economic/environment unit commitment with renewables and plug-in vehicles," Science China-Information Science, vol. 62, Article ID 070213, 2019. 